\title{
Symmetries and vacuum structure inside the Aoki phase
}

\author{
Vicente Azcoiti \\ Universidad de Zaragoza \\ E-mail: azcoitieazcoiti.unizar.es
}

\section{Giuseppe Di Carlo}

Laboratorio Nazionale di Gran Sasso, INFN

E-mail: gdicarlo@lngs.infn.it

\section{Eduardo Follana}

Universidad de Zaragoza

E-mail: efollana@unizar.es

\section{Alejandro Vaquero*}

CaSToRC, The Cyprus Institute

E-mail: a.vaquero@cyi.ac.cy

\begin{abstract}
After performing an analysis of the complete P.D.F. (probability distribution function) of several interesting operators inside the Aoki phase, we find strong indications that the $P^{\prime}$ symmetry (combination of Parity and a discrete flavor transformation) which forces $i \bar{\psi} \gamma_{5} \psi$ to be zero, is spontaneously broken. This result suggests a more complicated vacuum structure for the Aoki phase,contradicting the original $\chi P T$ predictions.
\end{abstract}

The XXIX International Symposium on Lattice Field Theory - Lattice 2011

July 10-16, 2011

Squaw Valley, Lake Tahoe, California

*Speaker. 


\section{Introduction}

We have performed research on the vacuum structure of the Aoki phase, by using the P.D.F. formalism for fermionic bilinears to predict the value of certain order parameters of symmetries. The P.D.F. formalism for fermionic bilinears [1] relates the spectrum of the Wilson Dirac operator in the Gibbs state and in the thermodynamic limit, to the expectation value of any power of any fermionic bilinear. Taking into account that the interesting order parameters of the symmetries we want to analyse inside the Aoki phase are fermionic bilinears, this method becomes the right choice. However, the P.D.F. is unable to predict the distribution of the eigenvalues of the Wilson Dirac operator, thence, we must measure this distribution numerically.

The fact that we measure quantities in the Gibbs state rise a problem: since the Gibbs state comprises all the possible existing vacua, in case of spontaneous symmetry breaking the mean value of any fermionic bilinear over the Gibbs state (taking into account all the vacua) will be zero, even if it is an order parameter of a broken symmetry

$$
\langle O\rangle=\sum_{\alpha} w_{\alpha}\langle O\rangle_{\alpha}=0
$$

where $\alpha$ indicates the vacuum where the observables are measured, $w_{\alpha}$ is the weight of that particular vacuum in the partition function, and $\langle O\rangle_{\alpha}$ is the expectation value of the operator $O$ in the vacuum $\alpha$. As the vacua related by the broken symmetry transformations have the same weights, all the expectation values cancel in the end and the average in the Gibbs state is zero. A simple way to solve this problem is to compute the expectation value of an even power of the operators, which should be non-zero if the symmetry is spontaneously broken.

The expected vacuum structure of the Aoki phase [2] is the following: both Parity $Z_{2}$ and flavour $S U$ (2) (in our two-flavoured case) symmetries are spontaneously broken to a $U(1)$ subgroup of flavour, and $\left\langle\left(i \bar{\psi} \gamma_{5} \tau_{3} \psi\right)^{2}\right\rangle$ becomes non-zero, but strangely $\left\langle\left(i \bar{\psi} \gamma_{5} \psi\right)^{2}\right\rangle$ vanishes, in spite of being a Parity order parameter. The explanation for this phenomenon is the existence of a 'modified Parity symmetry', called $P^{\prime}$, composed by a discrete flavour rotation plus Parity. According to the standard picture of the Aoki phase [3], this symmetry is the responsible of the vanishing expectation value of any even power of the bilinear $i \bar{\psi} \gamma_{5} \psi$ in the Gibbs state ${ }^{1}$. This picture was confirmed by $\chi P T$ calculations [3], which could describe the Aoki phase and its properties.

Maybe the best way to understand the subtle problems arising in this picture is to analyse the evolution of the expectation values of the fermionic bilinears we are interested in, $\left\langle\left(i \bar{\psi} \gamma_{5} \psi\right)^{2}\right\rangle$ and $\left\langle\left(i \bar{\psi} \gamma_{5} \tau_{3} \psi\right)^{2}\right\rangle$ from the physical phase to the critical line and beyond, inside the Aoki phase.

\section{The problems of the standard picture}

First of all one must recall the behaviour of the pions and of the $\eta$ meson as we approach the critical line from the "physical phase". These behaviours are related to the susceptibilities

$$
\chi_{\pi^{0}}=V\left[\left\langle\left(i \bar{\psi} \gamma_{5} \tau_{3} \psi\right)^{2}\right\rangle_{\alpha}-\left\langle i \bar{\psi} \gamma_{5} \tau_{3} \psi\right\rangle_{\alpha}^{2}\right]=V\left\langle\left(i \bar{\psi} \gamma_{5} \tau_{3} \psi\right)^{2}\right\rangle=\frac{2}{V}\left\langle\sum_{i=1}^{V} \frac{1}{\lambda_{i}^{2}}\right\rangle
$$

\footnotetext{
${ }^{1}$ Odd powers automatically vanish for symmetry reasons.
} 


$$
\chi_{\eta}=V\left[\left\langle\left(i \bar{\psi} \gamma_{5} \psi\right)^{2}\right\rangle_{\alpha}-\left\langle i \bar{\psi} \gamma_{5} \psi\right\rangle_{\alpha}^{2}\right]=V\left\langle\left(i \bar{\psi} \gamma_{5} \psi\right)^{2}\right\rangle=\frac{2}{V}\left\langle\sum_{i=1}^{V} \frac{1}{\lambda_{i}^{2}}\right\rangle-\frac{4}{V}\left\langle\left[\sum_{i=1}^{V} \frac{1}{\lambda_{i}}\right]^{2}\right\rangle
$$

where the $\lambda_{i}$ are the eigenvalues of the hermitian Dirac Wilson operator $\gamma_{5} D$, and the rightmost hand side of these equations is the P.D.F. expression for the expectation values of these operators, multiplied by the corresponding volume factor. Since outside the Aoki phase there exists only one vacuum, the Gibbs state expectation values (without subscript) can be identified with the $\alpha$-subscripted expectation values.

The first susceptibility should diverge at the critical line, so the neutral pion become massless. Moving beyond the critical line takes us to the Aoki phase, where it is well known the fact that

$$
\left\langle\left(i \bar{\psi} \gamma_{5} \tau_{3} \psi\right)^{2}\right\rangle=\frac{2}{V^{2}}\left\langle\sum_{i=1}^{V} \frac{1}{\lambda_{i}^{2}}\right\rangle \neq 0
$$

Notice the extra $1 / V$ factor with respect to eq. 2.1. Indeed, the bilinear $i \bar{\psi} \gamma_{5} \tau_{3} \psi$ is an order parameter for Parity-Flavour breaking, and therefore a landmark for the Aoki phase.

The $\eta$ meson, in contrast, behaves in a very different way, for due to the anomaly, it stays massive even at the critical line. Indeed the second contribution to the $\eta$-susceptibility, $\chi_{\eta}$, in 2.2 should diverge slower than $V$ at the critical line in order to compensate the divergency of the pion susceptibility (first contribution in 2.2) keeping this way a finite $\chi_{\eta}$. If we move deeper in the Aoki phase, it seems plausible to have a stronger divergency (as $V$ ) in such a way to allow the realization of the following equation

$$
\left\langle\left(i \bar{\psi} \gamma_{5} \psi\right)^{2}\right\rangle=\frac{2}{V^{2}}\left\langle\sum_{i=1}^{V} \frac{1}{\lambda_{i}^{2}}\right\rangle-\frac{4}{V^{2}}\left\langle\left(\sum_{i=1}^{V} \frac{1}{\lambda_{i}}\right)^{2}\right\rangle=0
$$

and thus realizing the standard picture of the Aoki phase. One might ask how can $i \bar{\psi} \gamma_{5} \psi$ be exactly zero in a Parity broken phase, but then the $P^{\prime}$ symmetry is introduced [3], and since $i \bar{\psi} \gamma_{5} \psi$ is also an order parameter for $P^{\prime}$, then it must vanish if the Aoki phase preserves the $P^{\prime}$ symmetry.

In fact, the effects of this $P^{\prime}$ symmetry are deeper. Since the standard picture of the Aoki phase requires $\left\langle i \bar{\psi} \gamma_{5} \psi\right\rangle_{\alpha}=0$ for any possible vacua, this can be translated in the P.D.F. language into the following statement: any even power of the bilinear $i \bar{\psi} \gamma_{5} \psi$ must have a vanishing expectation value in the Gibbs state. This statement, in the P.D.F. language, implies an infinite set of independent sum rules for the eigenvalues of the Dirac operator [4], each sum rule deriving from a different even power of the operator,

$$
\left\langle\left(i \bar{\psi} \gamma_{5} \psi\right)^{2 n}\right\rangle=0, \quad n=1,2,3 \ldots
$$

This tower of sum rules needn't be something exotical, and they can be enforced naturally by the realization of the $P^{\prime}$ symmetry $[3,5]$.

Up to now everything in the standard picture of the Aoki phase seems consistent. Now let's look at the operator

$$
Q=\frac{1}{V} \operatorname{Tr}\left[\left(\gamma_{5} D\right)^{-1}\right]=\frac{1}{V} \sum_{i=1}^{V} \frac{1}{\lambda_{i}}
$$


This quantity. which appears in the second piece of the r.h.s. of eq. 2.4, is an order parameter for the $P^{\prime}$ symmetry as well, and therefore it must vanish. Providing that it is an intensive operator, all its higher moments should also vanish in the Aoki phase if $P^{\prime}$ symmetry is realized in all vacua. But in this case $\left\langle\left(i \bar{\psi} \gamma_{5} \psi\right)^{2}\right\rangle$ can not vanish, for its first piece (see eq. 2.4) is known to be non-zero inside the Aoki phase. Hence we have a contradiction, and $P^{\prime}$ must be broken inside the Aoki phase. Nonetheless, without $P^{\prime}$ there is no way to explain the tower of sum rules, or even the vanishing of $\left\langle i \bar{\psi} \gamma_{5} \psi\right\rangle_{\alpha}$ in every possible vacua $\alpha$. The only way out for the standard picture is that the operator $Q$ be non-intensive. Alternatively, the standard picture of the Aoki phase maybe incomplete, and there might exist some new properties that have been overlooked during the past years.

What we propose $[4,6]$ is the addition of new vacua to the Aoki phase. We keep the vacua associated to the standard picture of the Aoki phase, but we add new ones, in order to remove the contradiction that arised around the $P^{\prime}$ symmetry. In these new vacua, the bilinear $i \bar{\psi} \gamma_{5} \psi$ is free to take non-vanishing values, Parity and $P^{\prime}$ are spontaneously broken, and there is no need of an infinite tower of sum rules.

\section{The simulations}

We decided to carry out HMC simulations of QCD with two flavours of Wilson fermions, inside and outside the Aoki phase, and without external sources, to find out $(a)$ whether the $P^{\prime}$ symmetry was preserved or not and $(b)$ the behaviour of the the P.D.F. of the operator $Q$ with the volume. Technical data of these simulations will be reported in a forcomming publication.

Unfortunately there is a problem with these simulations: since quasi-zero modes appear inside the Aoki phase, the eigenvalues are tempted to cross the origin, and they would do so, were it not for the fact that the crossing of eigenvalues is forbidden by the HMC dynamics due to the apparition of an infinite repulsion when very close to the origin. In order to solve this problem we classified our simulations according to its sector number, i.e., the number of 'crossed' eigenvalues they had beginning from a completely symmetric state (same number of positive and negative eigenvalues). Then we computed the weight of each sector in the partition function by simulating the same system, but with several values of the twisted mass term, and then extrapolating it to the case without external source (see Table 1).

\begin{tabular}{|c|c|c|c|c|c|}
\hline Volume & $m_{t}$ & Sector 0 & Sector 1 & Sector 2 & Sector 3+ \\
\hline $4^{4}$ & $0.0^{\star}$ & $67.65 \% \pm 1.29 \%$ & $32.23 \% \pm 1.35 \%$ & $0.11 \% \pm 0.07 \%$ & $0 \%$ \\
$4^{4}$ & 0.01 & $68.13 \% \pm 0.95 \%$ & $31.63 \% \pm 0.96 \%$ & $0.24 \% \pm 0.08 \%$ & $0 \%$ \\
$4^{4}$ & 0.10 & $69.28 \% \pm 0.90 \%$ & $30.44 \% \pm 0.89 \%$ & $0.28 \% \pm 0.04 \%$ & $0 \%$ \\
$4^{4}$ & Quench & $71.43 \% \pm 0.25 \%$ & $28.50 \% \pm 0.24 \%$ & $0.07 \% \pm 0.02 \%$ & $0 \%$ \\
$6^{4}$ & 0.10 & $51.41 \% \pm 0.64 \%$ & $44.67 \% \pm 0.64 \%$ & $3.86 \% \pm 0.07 \%$ & $0.06 \% \pm 0.02 \%$ \\
$6^{4}$ & Quench & $55.00 \% \pm 1.55 \%$ & $43.00 \% \pm 1.94 \%$ & $2.00 \% \pm 0.40 \%$ & $0 \%$ \\
\hline
\end{tabular}

Table 1: Weights of the different sectors as a function of the volume and $m_{t}$. The value marked with * was obtained from reweighting of the configurations generated at $m_{t}=0.01$.

This way we could reconstruct the P.D.F. for the interesting bilinears by summing the weighted P.D.F.'s of all sectors for a given volume. 


\section{Simulation results}

First of all, we checked the behaviour of the P.D.F. of the operator $Q$ outside the Aoki phase (point at $\beta=4.0$ and $\kappa=0.18$ ) in the three volumes considered $\left(4^{4}, 6^{4}\right.$ and $8^{4}$ ). As the left plot of fig. 1 clearly indicates, the operator $Q$ behaves as an intensive operator, and its P.D.F. tends to a Dirac delta in the origin as the volume increases, so all of its moments vanish in the thermodynamic limit.
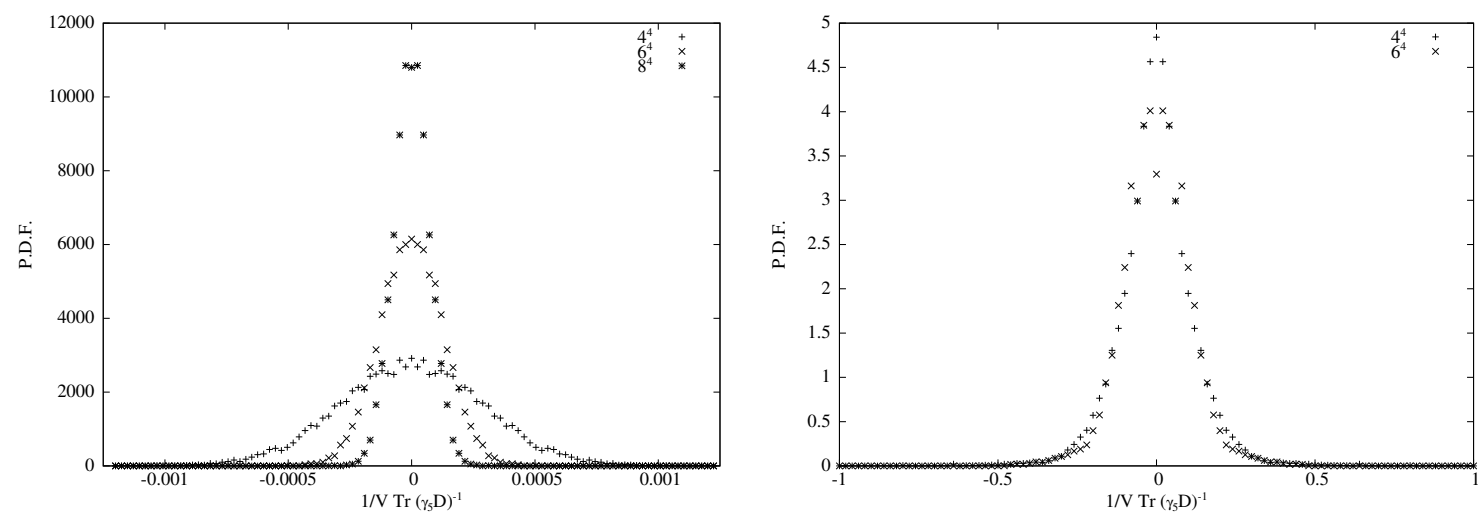

Figure 1: On the left, the P.D.F. of $Q$ outside the Aoki phase. On the center, the P.D.F. of $Q$ inside the Aoki phase. The behaviour points clearly to SSB. On the right, the P.D.F. of $Q$ obtained from configurations generated with a twisted mass term.

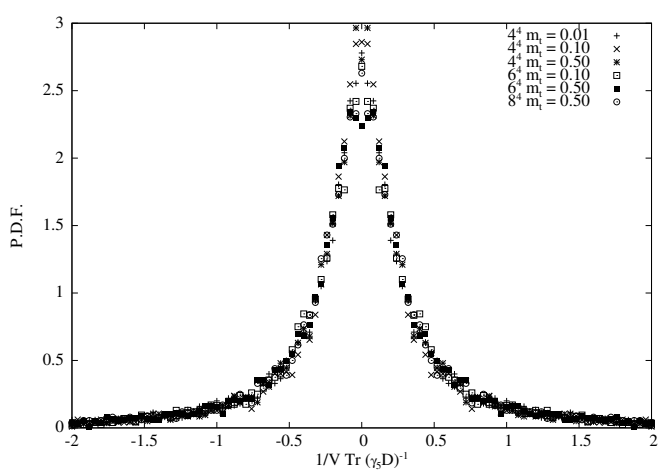

Figure 2: The P.D.F. of $Q$ obtained from configurations generated with a twisted mass term.

With this simple result we must conclude that there is a subtle problem in the standard formulation of the Aoki phase, but we can go further. Let's see what happens inside the Aoki phase ( $\beta=2.0$ and $\kappa=0.25^{2}$ ), and for this case we will use data from the $4^{4}$ and $6^{4}$ volumes (the $8^{4}$ inside the Aoki phase was very expensive for us). We show in the right plot of fig. 1 how the two volumes converge to a single peak of constant width, therefore we expect the $P^{\prime}$ symmetry to be spontaneously broken inside the Aoki phase. Without a $P^{\prime}$ symmetry behind, the realization of

\footnotetext{
${ }^{2}$ During the conference people were concerned that, at such a low $\beta$, we were far from the continuum limit, and then it didn't make sense to speak about SSB. Nonetheless our analysis is devoted to the Aoki phase on the lattice, which might even not have a continuum limit. Thence we have to look at this system as an statistical system with Wilson fermions, not as QCD. In this context, it is perfectly valid to speak about SSB.
} 


\begin{tabular}{|c|c|c|c|}
\hline Volume & $\left\langle\left(i \bar{\psi}_{u} \gamma_{5} \psi_{u}\right)^{2}\right\rangle$ & $\left\langle\left(i \bar{\psi} \gamma_{5} \psi\right)^{2}\right\rangle$ & $\left\langle\left(i \bar{\psi} \gamma_{5} \tau_{3} \psi\right)^{2}\right\rangle$ \\
\hline $4^{4}$ & $(1.495 \pm 0.037) 10^{-2}$ & $(3.7 \pm 1.9) 10^{-3}$ & $(5.60 \pm 0.16) 10^{-2}$ \\
$6^{4}$ & $(1.625 \pm 0.026) 10^{-2}$ & $(1.098 \pm 0.098) 10^{-2}$ & $(5.402 \pm 0.067) 10^{-2}$ \\
\hline
\end{tabular}

Table 2: Interesting v.e.v. for the Aoki phase

the infinite tower of sum rules becomes quite enigmatic. Of course we are far from the thermodynamical limit, and one might argue that, as stated in table 1, the sector 2 contribution for the $6^{4}$ volume is important enough to be taken into account. This sector was not simulated because it was extremely expensive from the numerical point of view, and for sure it will modify the final results shown here, but since its weight is less than 5\%, we don't expect these changes to be relevant for the final result.

In order to reinforce the statement that there are new unconsidered vacua inside the Aoki phase, we can measure the P.D.F. of the operator $Q$ in configurations generated with several values of an external Aoki-like source term, $h i \bar{\psi} \gamma_{5} \tau_{3} \psi$, but assuming that those are the configurations of the Gibbs state. Since the addition of an external source selects a standard Aoki vacuum, we expect that all the contributions to the P.D.F. coming from the other vacua will be removed. As we can see in fig. 2, the obtained P.D.F. for this case is essentially different than the one shown in the right plot of fig. 1: the P.D.F. seems independent of the value of the external field and of the volume, but when we compare this distribution with the former one in the right of fig. 1, we notice that the P.D.F. of the operator with external source is much wider ${ }^{3}$, and since the P.D.F. depends heavily on the spectrum of the Dirac operator, we expect the spectrum to be different as well.

In contrast, if in the computation of the P.D.F. we take into account the fact that we are using configurations generated with an external source term, then the P.D.F. expressions change accordingly, and we find that the final P.D.F. of the operator $Q$ is a Dirac delta at the origin, a result quite expected from all the existent work on the Aoki phase, including chiral lagrangians.

Since $P^{\prime}$ is broken according to our results, the arguments supporting the existence of a tower of sum rules are no longer valid, thus it is natural to think that the expectation value $\left\langle\left(i \bar{\psi} \gamma_{5} \psi\right)^{2}\right\rangle$ will be non-zero in the Gibbs state inside the Aoki phase. As stated in former papers [6] on the subject, this observable is extremely difficult to measure, nonetheless we managed to obtain sensible results from our data, which we show in Table 2.

The last column refers to $\left\langle\left(i \bar{\psi} \gamma_{5} \tau_{3} \psi\right)^{2}\right\rangle$, the landmark of the Aoki phase. As we see, it is clearly non-zero for all the volumes, confirming that our simulations lie within the Aoki phase. The first column belongs to $\left\langle\left(i \bar{\psi}_{u} \gamma_{5} \psi_{u}\right)^{2}\right\rangle$, which is an order parameter for Parity, but it takes into account just one flavour (which we labeled $u$ ). This quantity should be non-zero inside the Paritybreaking Aoki phase, regardless of the discussion of the new vacua. Finally, the most important observable, the flavour singlet pseudoscalar $\left\langle\left(i \bar{\psi} \gamma_{5} \psi\right)^{2}\right\rangle$, which -since we are dealing with two degenerated flavours, $u$ and $d$ - is the sum of two of the former condensates $i \bar{\psi}_{u} \gamma_{5} \psi_{u}+i \bar{\psi}_{d} \gamma_{5} \psi_{d}$. The standard picture of the Aoki phase predicts zero expectation value of this parameter in any Aoki

\footnotetext{
${ }^{3}$ The reason can be deduced from the properties of the spectrum: in the case with external source, there is no lower bound for the modulus of an eigenvalue, but in the case without external source, the lower bound is given by $1 / V$. This difference generates the long tails of the case with an external source.
} 
vacua, whereas each one of the pseudoscalars restricted to one flavour $i \bar{\psi}_{u, d} \gamma_{5} \psi_{u, d}$ will not vanish due to Parity breaking. Hence, the standard picture of the Aoki phase enforces an antiferromagnetic ordering of the pseudoscalars $i \bar{\psi}_{u} \gamma_{5} \psi_{u}=-i \bar{\psi}_{d} \gamma_{5} \psi_{d}$, which is not require in our hypothesis of the new vacua. The data in table 2 shows a clear non-zero expectation value for $\left\langle\left(i \bar{\psi} \gamma_{5} \psi\right)^{2}\right\rangle$ in the case of the largest volume $6^{4}$, of the same order of magnitude as $\left\langle\left(i \bar{\psi}_{u} \gamma_{5} \psi_{u}\right)^{2}\right\rangle$, supporting our previous discussion regarding $P^{\prime}$ breaking.

\section{Conclusions}

During the last years we have performed an extensive research to find out if our alternative vacuum structure of the Aoki phase, derived from a P.D.F. analysis, was realized or not. For the first time we can provide some numerical evidence of the realization of the new vacua, and of $P^{\prime}$ symmetry breaking by demonstrating that the P.D.F. of the operator $Q$, an order parameter for the $P^{\prime}$ symmetry, is not a Dirac delta inside the Aoki phase. On the other hand, as $P^{\prime}$ is required to explain the current picture of the Aoki phase, our result calls into question the validity of the standard Aoki picture. We were also able to perform a direct measurement of $\left\langle\left(i \bar{\psi} \gamma_{5} \psi\right)^{2}\right\rangle$, which casted a non-zero value. Were this result also true in the thermodynamic limit, it would break the hypothesis of the sum-rules. As the sum-rules are a direct consequence of the $\chi P T$ application to the Aoki phase, we are also calling into question the validity of the $\chi P T$ analysis performed in the articles [3] for low values of $\beta$ (around 2.0, very coarse lattices). Since $\chi P T$ is expected to work well at higher values of $\beta$, there might be a way to reconcile the two different views, for the terms in the Symanzik expansion of the action are proportional to powers of the lattice spacing $a$, and may it happen that at such a low $\beta$ as 2.0 , we need more terms in the $\chi P T$ lagrangian to describe properly the theory. In any case, should we confirm our alternative vacuum structure for the Aoki phase for higher volumes, the $\chi P T$ results for this scenario should be revised.

\section{Acknowledgements}

This work was funded by an INFN-MICINN collaboration, MICINN (under grants FPA200909638 and FPA2008-10732), DGIID-DGA (grant 2007-E24/2), and by the EU under ITN-STRONGnet (PITN-GA-2009-238353). E. Follana is supported on the MICINN Ramon y Cajal program.

\section{References}

[1] V. Azcoiti, V. Laliena and X.Q. Luo, Phys. Lett. B 354, (1995) 111.

[2] S. Aoki, Phys. Rev. D 30, (1984) 2653; Phys. Rev. Lett. 57, (1986) 3136.

[3] S. Sharpe and R. Singleton, Jr., Phys. Rev. D 58, (1998) 074501 [hep-lat/9804028]; Nucl. Phys. Proc. Suppl. 73, (1999) 234 [hep-lat/9808047].

[4] V. Azcoiti, G. di Carlo and A. Vaquero, Phys. Rev. D 79, (2009) 014509 [arXiv: 0809.2972 ].

[5] S. Sharpe, Phys. Rev. D 79, (2009) 054503 [arXiv: 0811.0409 ].

[6] V. Azcoiti, G. di Carlo, E. Follana and A. Vaquero, PoS(Lattice 2009) (2009), 068 [arXiv:0910 .0947]; PoS(Lattice 2010) (2010), 091 [arXiv:1012.2781]. 